\title{
Allergic Reactions to Metamizole: Immediate and Delayed Responses
}

\author{
Natalia Blanca-López ${ }^{a} \quad$ Natalia Pérez-Sánchez ${ }^{b} \quad$ José Augusto Agúndez ${ }^{d}$ \\ Elena García-Martin ${ }^{d}$ María José Torres ${ }^{b}$ José Antonio Cornejo-Garcíac \\ James R. Perkins ${ }^{c}$ Miguel Angel Miranda ${ }^{e}$ Inmaculada Andreu ${ }^{e}$ \\ Cristobalina Mayorgac Gabriela Canto ${ }^{a}$ Miguel Blancab Inmaculada Doñab \\ ${ }^{a}$ Allergy Service, Infanta Leonor Hospital, Madrid, b Allergy Unit and ${ }^{c}$ Research Laboratory, IBIMA, Regional \\ University Hospital of Malaga, UMA, Malaga, dDepartment of Pharmacology, University of Extremadura, Caceres, \\ and ${ }^{\mathrm{e} C h e m i c a l}$ Technology Institute, UPV-CSIC, Polytechnic University of Valencia, Valencia, Spain
}

\section{Key Words}

Basophil activation test - Drug provocation test .

Metamizole $\cdot$ Selective hypersensitivity $\cdot$ Skin test

\begin{abstract}
Background: Pyrazolones are the most common causes of selective nonsteroidal anti-inflammatory drug (NSAID) hypersensitivity. We studied a large group of patients with immediate and delayed selective responses to metamizole. Methods: Patients with suspicion of hypersensitivity to metamizole were evaluated. We verified acetylsalicylic acid tolerance and classified patients as immediate or delayed responders if they showed symptoms less or more than $24 \mathrm{~h}$ after metamizole administration. Skin tests were performed and if negative, a basophil activation test (BAT) was performed on immediate responders. If it was negative, we performed a drug provocation test (DPT) with metamizole. $\boldsymbol{R e}$ sults: A total of 137 patients were included: 132 reacted within $24 \mathrm{~h}$ (single NSAID-induced urticaria/angioedema/ anaphylaxis; SNIUAA) and 5 after $24 \mathrm{~h}$ (single NSAID-induced delayed hypersensitivity reaction; SNIDHR). Most SNIUAA patients developed anaphylaxis (60.60\%); for SNIDHR, maculopapular exanthema was the most frequent entity (60\%). Skin testing was positive in $62.04 \%$ of all cases and BAT in
\end{abstract}

$28 \%$ of the SNIUAA patients with negative skin tests. In $5.1 \%$ of the cases, DPT with metamizole was needed to establish the diagnosis. In $22.62 \%$ of the cases, diagnosis was established by consistent and unequivocal history of repeated allergic episodes in spite of a negative skin test and BAT. Conclusions: SNIUAA to metamizole is the most frequent type of selective NSAID hypersensitivity, with anaphylaxis being the most common clinical entity. It may occur within $1 \mathrm{~h}$ after drug intake. SNIDHR occurs in a very low percentage of cases. The low sensitivity of diagnostic tests may be due to incomplete characterization of the chemical structures of metamizole and its metabolites.

ㄷ) 2016 S. Karger AG, Basel

\section{Introduction}

Adverse drug reactions constitute an important public health issue, causing 3-6\% of all hospital admissions and occurring in $10-15 \%$ of hospitalized patients [1]. Nonsteroidal anti-inflammatory drugs (NSAIDs) are the most frequent medicines involved in drug hypersensitivity reactions in both adults [2] and children [3] followed by $\beta$-lactam antibiotics [4]. Hypersensitivity reactions to NSAIDs have been classified into different categories de-

\section{KARGER}

E-Mail karger@karger.com

www.karger.com/iaa (c) 2016 S. Karger AG, Basel

$1018-2438 / 16 / 1694-0223 \$ 39.50 / 0$
Correspondence to: Dr. Inmaculada Doña

Allergy Unit, IBIMA, Regional University Hospital of Malaga, UMA

Plaza del Hospital Civil s/n

ES-29009 Malaga (Spain)

E-Mail inmadd@ hotmail.com 
pending on the clinical symptoms induced, the number of NSAIDs involved and the presence or absence of underlying disease [5]. The following classification has been proposed: (1) NSAID-exacerbated respiratory disease; (2) NSAID-exacerbated cutaneous disease; (3) NSAIDinduced urticaria/angioedema; (4) single NSAID-induced urticaria/angioedema/anaphylaxis (SNIUAA), and (5) single NSAID-induced delayed hypersensitivity reaction (SNIDHR).

The mechanism involved in the first three reaction types is thought to be nonimmunologically mediated (cross hypersensitivity), but it is related to the inhibition of a cyclooxygenase enzyme (COX-1) [5]. The last two categories involve an immunologically mediated response that is induced by a single drug/drug group, with subjects tolerating other chemically unrelated compounds (selective response) including strong COX-1 inhibitors $[5,6]$. In SNIUAA, symptoms usually occur shortly after drug intake [5] and an IgE-mediated mechanism has been proposed [7-10]. In SNIDHR, reactions occur 24-48 h or longer after drug intake [5] and a T cellmediated mechanism is likely [11]. As occurs with $\beta$-lactam antibiotics, symptoms may appear at a shorter interval after drug intake $[12,13]$.

Most studies of hypersensitivity reactions to NSAIDs have focused on nonimmunologically mediated reactions (cross hypersensitivity) [14-17], mainly in NSAID-exacerbated respiratory disease, although there is growing interest in the cutaneous entities (NSAID-induced urticaria/angioedema and NSAID-exacerbated cutaneous disease) [14-19]. Although immunologically mediated reactions account for $25-30 \%$ of all NSAID hypersensitivity reactions [20], less attention has been paid to these reactions and no studies have been performed looking at large series of well-phenotyped cases. It is known that pyrazolones, particularly metamizole $\{[\mathrm{N}$ - $(1,5$-dimethyl3-oxo-2-phenylpyrazolin-4-yl)-N-methylamino]methanesulfonate, drug bank identity No. DB04817\}, are the most frequent drugs involved in immunologically mediated reactions $[7,20,21]$. Their use is widespread in many countries due to their analgesic, antipyretic and spasmolytic properties, and therefore many patients are exposed.

Our aim was to study a large group of patients who developed selective responses (SR) to metamizole, one of the most frequently used analgesics in our population, and to establish in how many case responses were immediate or delayed, following the classification provided by the European Network for Drug Allergy Group [5]. The contribution of diagnostic tests (both in vivo and in vitro) was also assessed.

\section{Methods}

\section{Patients}

We evaluated patients with symptoms suggestive of hypersensitivity reactions to metamizole referred to the Allergy Unit of the University Regional Hospital of Málaga and the Infanta Leonor Hospital in Madrid over a period of 3 years (2012-2014).

Inclusion Criteria. Patients aged 14-80 years with a confirmed diagnosis of SR to metamizole were included. The diagnosis was established according to the algorithm shown in figure 1 . The first approach was to verify tolerance to acetylsalicylic acid (ASA) if this was not known. If subjects responded to ASA, they were considered cross hypersensitive to NSAIDs and not included in this study. If subjects tolerated ASA in a drug provocation test (DPT), they were considered as having either immediate reactions when they had the symptoms less than $24 \mathrm{~h}$ after metamizole administration or as delayed reactions when symptoms occurred more than $24 \mathrm{~h}$ later. Skin tests with metamizole were performed for patients with both immediate and delayed reactions, as described previously [22]. In patients with immediate reactions, if skin tests were negative, a basophil activation test (BAT) with metamizole was carried out. If skin tests or BAT were positive, the patients were confirmed as having SR to metamizole. If both skin test and BAT were negative, we considered the number of episodes suffered after metamizole administration: if the patient had at least 2 episodes, they were diagnosed as having SR to metamizole, but if the patient had only 1 episode, a positive DPT with metamizole was required, except in subjects with severe reactions (e.g. toxic epidermal necrolysis or anaphylactic shock).

Exclusion Criteria. We excluded patients younger than 14 years or older than 80 years of age; patients with a confirmed diagnosis of cross hypersensitivity to NSAIDs; patients with 1 reported prior reaction to metamizole, with negative skin test and BAT results, where DPT with metamizole was contraindicated; patients who tolerated metamizole; patients where DPT to COX-1 inhibitor is contraindicated due to underlying disease; pregnant or breastfeeding patients; patients taking $\beta$-blockers or ACE inhibitors or with contraindications to epinephrine administration; patients who had acute infections and/or underlying cardiac, hepatic or renal diseases that contraindicated DPT, and patients with psychosomatic disorders.

\section{Clinical History}

Patients were questioned about the symptoms induced by metamizole administration; the time interval between drug intake and reaction onset; the number of episodes; the time interval between the last reaction and study; underlying nasal and bronchial symptoms, food allergy and the presence of underlying chronic spontaneous urticaria, either active or in remission.

\section{Atopy Status Assessment}

The atopy status was assessed with a skin prick test (SPT) performed with a battery of 20 common inhalant allergens, including pollens, house dust mites, molds and animal danders, and a battery of 31 common food allergens that included animal, fruit and vegetable allergens (ALK, Madrid, Spain). Histamine hydrochloride $(10 \mathrm{mg} / \mathrm{ml})$ and phenolated glycerol saline were used as positive and negative controls, respectively. A positive SPT response was defined as a wheal diameter of $3 \mathrm{~mm}$ or larger to at least one of these allergens. The patients were requested to stop taking any 


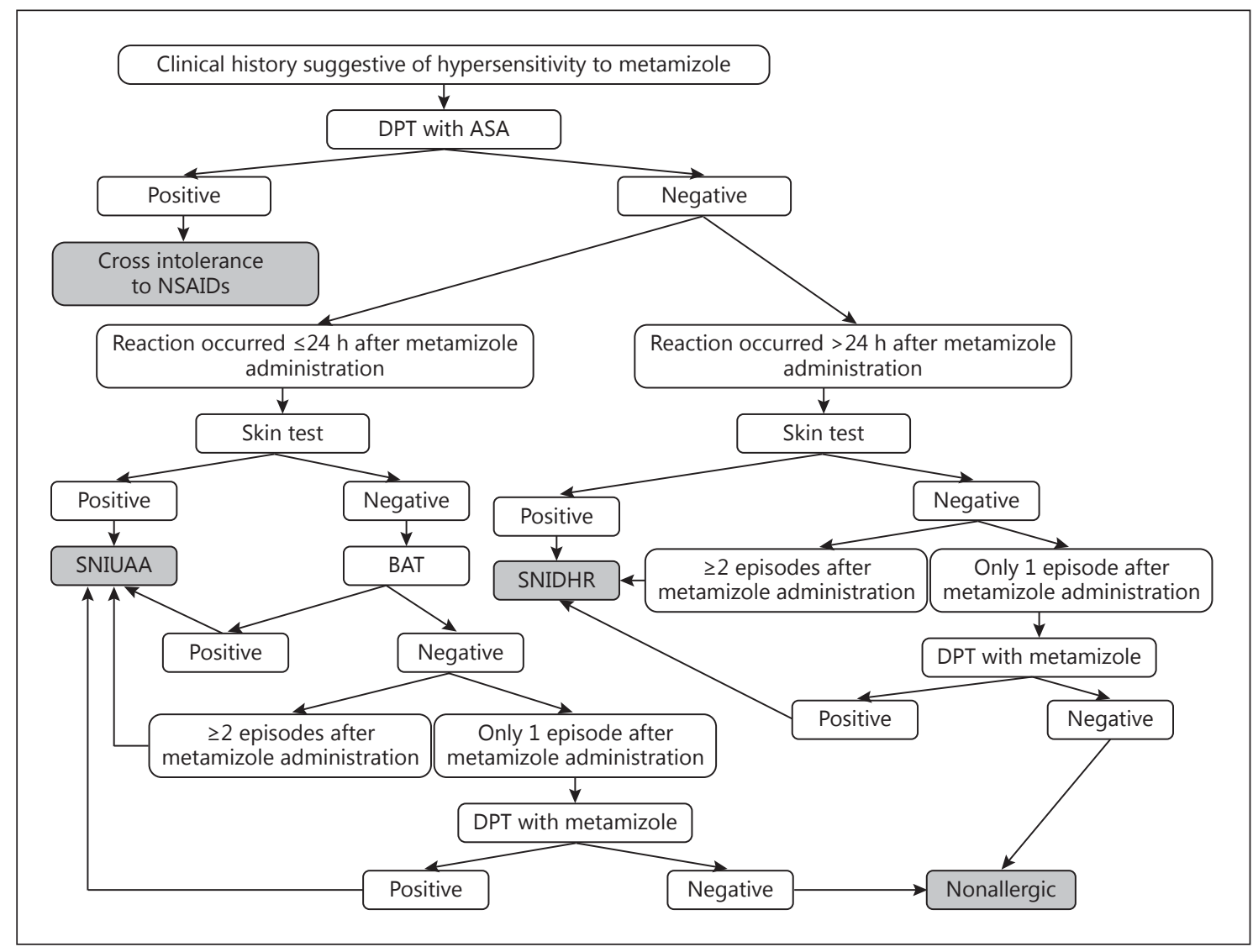

Fig. 1. Algorithm for the diagnosis of patients with reactions suggestive of hypersensitivity to metamizole.

medications that contained antihistamine at least 8 days before skin testing.

\section{Skin Testing}

For immediate reactions, SPT and intradermal test (ID) were carried out as described [22] using metamizole (Boehringer Ingelheim, Barcelona, Spain) at 40 and $400 \mathrm{mg} / \mathrm{ml}$ for SPT and at 0.4 and $4 \mathrm{mg} / \mathrm{ml}$ for ID. In cases reporting severe reactions, ID was initially performed using 0.004 and $0.04 \mathrm{mg} / \mathrm{ml}$. An increase in the diameter of the wheal by more than $3 \mathrm{~mm} 20 \mathrm{~min}$ after testing was considered positive for SNIUAA.

For delayed reactions, patch and ID tests were carried out and evaluated after $48 \mathrm{~h}$, as described [22]. For ID tests, the presence of intradermal papular induration after $48 \mathrm{~h}$ was considered positive. Patch tests were performed by mixing powdered metamizole in petrolatum at $10 \% \mathrm{w} / \mathrm{w}$. The occlusion time was $48 \mathrm{~h}$. Erythema with edema, papules, vesicles or bullae 48 and/or $72 \mathrm{~h}$ after testing was considered positive [22].

\section{Basophil Activation Test}

In patients with a suspected immediate reaction, BAT was performed as described [23] using metamizole (Boehringer Ingelheim) at 0.25 and $2.5 \mathrm{mg} / \mathrm{ml}$. Results were considered positive when the stimulation index, calculated as the ratio of the percentage of de- granulated basophils with the different haptens to the negative control, was greater than 2 in at least one of the concentrations used.

\section{Oral Drug Provocation Test}

In order to verify tolerance to a strong COX-1 inhibitor, DPT with ASA was performed in a single-blind manner, as described [20]: placebo capsules were given at different times on the 1st day, three doses of ASA were administered orally at intervals of $90 \mathrm{~min}$ (5, 30 and $100 \mathrm{mg}$ ) on the $2 \mathrm{nd}$ day, and, if negative, another two doses of ASA (150 and $300 \mathrm{mg}$ ) on the $3 \mathrm{rd}$ day. If a patient had only 1 episode after metamizole administration and no contraindications for DPT existed, increasing doses of metamizole were administered orally at intervals of 90 min for 2 days (1st day: 5, 10 and 50 mg; cumulative dose $65 \mathrm{mg}$; 2nd day: 50, 150 and $300 \mathrm{mg}$; cumulative dose $500 \mathrm{mg}$ ).

If cutaneous and/or respiratory symptoms or alterations in vital signs (rhythm alterations, decrease in peak expiratory flow or hypotension) appeared, the procedure was stopped and the symptoms were evaluated and treated. If no symptoms appeared during drug administration, the therapeutic dose of ASA/metamizole was achieved. If tolerance occurred, this was followed by 2 days $/ 8 \mathrm{~h}$ at maximum dose, after a gap of $24 \mathrm{~h}$. ASA, metamizole and placebo were given in opaque capsules prepared by the hospital pharmacy service. 
Table 1. Clinical data comparing SNIUAA and SNIDHR

\begin{tabular}{lll}
\hline & SNIUAA $(\mathrm{n}=132)$ & SNIDHR $(\mathrm{n}=5)$ \\
\hline $\begin{array}{l}\text { Age (IQR), years } \\
\text { Gender, n (\%) }\end{array}$ & $53(41.25-63)$ & $68(31.75-75.75)$ \\
$\quad$ Female & $97(73.48)$ & $4(80)$ \\
$\quad$ Male & $35(26.51)$ & $1(20)$ \\
Number of episodes reported after & $2(1-2)$ & $2(2-3)$ \\
$\quad$ metamizole administration & & \\
Clinical entities, n (\%) & $80(60.60)$ & 0 \\
$\quad$ Anaphylaxis & $42(31.81)$ & 0 \\
Urticaria & $7(5.3)$ & 0 \\
Angioedema & $1(0.75)$ & 0 \\
Glottis edema & $2(1.51$ & $3(60)$ \\
Fixed drug eruption & 0 & $1(20)$ \\
MPE & 0 & $1(20)$ \\
Exanthema with bullae & 0 & \\
Exanthema with skin desquamation & & \\
\hline
\end{tabular}

Forced expiratory volume in $1 \mathrm{~s}$ had to be at least $80 \%$ of predicted values, with an absolute value of at least 1.5 liters. Antihistamine agents were stopped 1 week before challenge.

\section{Statistical Analysis}

Data analysis was performed using $\chi^{2}$ analysis to test differences in nominal variables between groups, the Fisher test was used when there were no criteria for using the $\chi^{2}$ test and the Mann-Whitney test was used for quantitative variables. All reported $p$ values represented two-tailed tests, with values of $p<0.05$ considered statistically significant. The analysis included age, gender, atopic status, number of episodes, clinical manifestations and methods used for the diagnosis.

The study was conducted according to the principles of the Declaration of Helsinki and approved by the Ethics Committees of the University Regional Hospital of Malaga. All the participants were informed orally about the study and signed the corresponding informed consent.

\section{Results}

A total of 5,926 patients with a clinical history of drug hypersensitivity reactions were evaluated at the Allergy Units of the University Regional Hospital of Malaga and the Infanta Leonor Hospital in Madrid in 2012-2014. NSAIDs were involved in 2,398 cases. In 922 cases, metamizole was the NSAID involved in the episodes. Of these, a total of 137 patients were confirmed as having SR to metamizole and were included in this study. The remaining 785 patients with reactions after metamizole intake were not considered for this study due to cross hypersensitivity (678 subjects) or unconfirmed diagnosis
(107 subjects). Of these, 6 were pregnant; 101 had negative skin test and BAT, and could not undergo DPT to ASA and/or metamizole ( 40 were older than 70 years and had cardiopulmonary comorbidities, 41 reported anaphylactic shock and 20 severe delayed reactions).

The 137 patients with confirmed SR to metamizole included in this study had a median age of 53 years [interquartile range (IQR): 41-64] and 101 were women (73.72\%). Fifty-seven cases (41.6\%) were atopic and 35 (25.54\%) had rhinitis, 10 (7.29\%) had asthma, 10 (7.29\%) had symptoms attributed to food allergy and $7(5.1 \%)$ had underlying chronic urticaria.

Considering the total group and based on clinical history (table 1), most cases with confirmed SR to metamizole developed anaphylaxis $(80 ; 58.39 \%)$, followed by urticaria $(42,30.65 \%)$, angioedema $(7,5.1 \%)$, maculopapular exanthema (MPE; 3, 2.18\%), fixed drug eruption (2, $1.45 \%)$ and glottis edema, exanthema with bullae and exanthema with skin desquamation (only 1 patient each; $0.7 \%)$. Concerning the number of previously reported episodes, patients had a median of 2 (IQR: 1-2). Analyzing the time interval between metamizole administration and the onset of reactions reported in clinical history, in a total of $101(73.72 \%)$ patients the reaction occurred within $30 \mathrm{~min}$; in 13 patients $(9.48 \%)$ within $30-60 \mathrm{~min}$; in $9(6.56 \%)$ within $1-2 \mathrm{~h}$; in $9(6.56 \%)$ within $2-8 \mathrm{~h}$, and in $5(3.64 \%)$ more than $24 \mathrm{~h}$ later. For further analysis, we classified patients as SNIUAA if the time interval was less than $24 \mathrm{~h}$ after metamizole administration (132; 96.35\%) and SNIDHR if the interval was more than $24 \mathrm{~h}$ later (5; $3.64 \%)$. 
Table 2. Involvement of different organs and administration route of metamizole in patients who reported anaphylaxis

\begin{tabular}{lrc}
\hline Organ system involved & $\mathrm{n}(\%)$ & Route \\
\hline Skin + respiratory system & $24(30)$ & p.o. \\
Skin + gastrointestinal system & $2(2.5)$ & p.o. \\
Skin + cardiovascular system & $2(2.5)$ & p.o. \\
Skin + respiratory + gastrointestinal system & $5(6.25)$ & p.o. \\
Skin + transitory loss of consciousness & $22(27.5)$ & p.o. \\
Skin + respiratory system + transitory loss of consciousness & $9(11.25)$ & p.o. \\
Skin + gastrointestinal system + transitory loss of consciousness & $9(11.25)$ & p.o. \\
Skin + respiratory + gastrointestinal system + transitory loss of consciousness & $2(2.5)$ & p.o. \\
Skin + respiratory + cardiovascular system + transitory loss of consciousness & $3(3.75)$ & i.v. \\
Skin + respiratory + gastrointestinal + cardiovascular system & $2(2.5)$ & i.v. + p.o. ${ }^{1}$ \\
\hline
\end{tabular}

${ }^{1}$ The symptoms described for this group were those corresponding to the intravenous route.

Considering the patients with anaphylaxis $(\mathrm{n}=80)$, in all cases there was skin involvement. The participation of the other organs is listed in table 2 . The respiratory involvement consisted of dyspnea, wheezing and chest tightness, the gastrointestinal symptoms consisted of abdominal cramps, vomiting and diarrhea, and the cardiovascular symptoms consisted of tachycardia and hypotension.

An analysis of the time intervals in the cases of anaphylaxis revealed that in 70 patients $(87.5 \%)$ the reactions occurred in less than $30 \mathrm{~min}$, in $6(7.5 \%)$ between 30 and 60 min, in $2(2.5 \%)$ between 1 and $2 \mathrm{~h}$ and in $2(2.5 \%)$ between 2 and $8 \mathrm{~h}$. No cases of anaphylaxis occurred beyond this time.

According to clinical history, most cases reported to have taken metamizole via the oral route and 5 via the intravenous route. In 2 patients of this group, there was also another episode after oral intake. In these cases, the reactions reported were more severe via the intravenous route, involving four organs (skin and respiratory, cardiovascular and gastrointestinal system or transitory loss of consciousness), compared with administration via the oral route (table 2 ). In this 5 cases, the symptoms presented within $30 \mathrm{~min}$.

No differences were found in age, gender, atopy, rhinitis, asthma, food allergy, underlying chronic urticaria and number of episodes reported when comparing SNIUAA and SNIDHR.

Most SNIUAA patients $(80 ; 60.60 \%)$ had anaphylaxis whilst amongst SNIDHR patients the most frequent clinical entity was MPE $(3 ; 60 \%)$. The median time interval
Table 3. Methods used for SNIUAA and SNIDHR diagnosis

\begin{tabular}{lll}
\hline Methods for diagnosis & $\begin{array}{l}\text { SNIUAA, } \mathrm{n} \\
(\mathrm{n}=132)\end{array}$ & $\begin{array}{l}\text { SNIDHR, } \mathrm{n} \\
(\mathrm{n}=5)\end{array}$ \\
\hline Skin test & $37(28.03 \%)$ & Not done \\
$\quad$ SPT & $45(47.36 \%)$ & $3(60 \%)$ \\
ID & Not done & $3(60 \%)$ \\
$\quad$ Patch & $14(28 \%)^{\mathrm{a}}$ & Not done \\
BAT & $6(12 \%)^{\mathrm{a}}$ & $1(20 \%)$ \\
DPT with metamizole & $30(60 \%)^{\mathrm{a}}$ & $1(20 \%)$ \\
Clinical history + DPT ASA & & \\
\hline
\end{tabular}

${ }^{a}$ Of 50 patients with a negative skin test.

between the last reaction and the study was 6 months (IQR: 3-24). No differences were found between SNIUAA and SNIDHR.

Of the 137 cases evaluated, 85 (62.04\%) patients were positive to skin tests (table 3). For SNIUAA, 37 (28.03\%) were SPT positive and $45(47.36 \%)$ were ID positive. For SNIDHR, 3 (60\%) were positive to both ID and patch test (table 2). One patient developed an immediate systemic response during SPT with metamizole, although the reading was negative. In SNIUAA patients with negative skin test results $(n=50)$, BAT with metamizole was performed and positive in 14 subjects (28\%).

Comparing patients with positive and negative results in skin tests and BAT, the time interval between the last reaction induced by metamizole and the study was short- 
Table 4. Clinical data of the patients with DPT to metamizole

\begin{tabular}{|c|c|c|c|c|c|}
\hline $\begin{array}{l}\text { Patient } \\
\text { No. }\end{array}$ & $\begin{array}{l}\text { Age, years/ } \\
\text { gender }\end{array}$ & Clinical entity & TIR & $\begin{array}{l}\text { Dose, } \\
\mathrm{mg}\end{array}$ & Symptoms \\
\hline 1 & $46 / \mathrm{F}$ & Urticaria & 30 & 65 & Generalized pruritus and facial angioedema \\
\hline 2 & $41 / \mathrm{F}$ & Urticaria + angioedema & 45 & 575 & Pruritus (hands) and wheals (thorax and abdomen) \\
\hline 3 & $42 / \mathrm{F}$ & Urticaria & 60 & 205 & Systemic pruritus, conjunctival injection and tongue edema \\
\hline 4 & $33 / \mathrm{M}$ & Urticaria + angioedema & 30 & 575 & Wheals (abdomen) plus pruritus \\
\hline 5 & $43 / \mathrm{M}$ & Urticaria & 45 & 65 & Pruritus (thorax, arms and back) and wheals (thorax) \\
\hline 7 & $67 / \mathrm{M}$ & MPE & 2,880 & 575 & MPE of the trunk \\
\hline
\end{tabular}

TIR = Time interval between metamizole administration and the reactions $(\mathrm{min})$.

er in those who had positive tests [3 (IQR: 3-12) vs. 12 (IQR: 3-36) months, $\mathrm{p}=0.023$ ].

The results of DPT with metamizole are shown in table 4. A total of 6 cases who reported immediate reactions after metamizole administration had negative skin tests and BAT and only 1 episode induced by metamizole; 1 case who reported a delayed reaction had negative skin tests and only had 1 episode induced by metamizole. In all cases, DPT with metamizole induced mild symptoms: 7 patients developed pruritus and wheals localized on different parts of the body and 1 MPE with no systemic symptoms. No patient had respiratory or cardiovascular system involvement. The patients responded to a median dose of $480 \mathrm{mg}$ (IQR: 65-575) of metamizole. The symptoms disappeared within 1-48 $\mathrm{h}$ of administering antihistamine and corticosteroid treatment.

In 31 patients $(22.62 \%)$ with both negative skin tests and BAT, the diagnosis was achieved by clinical history as they had 2 or more episodes induced by metamizole and tolerance to ASA was confirmed by DPT (table 3 ).

\section{Discussion}

We have evaluated a large group of cases with hypersensitivity to pyrazolones following the consensus guidelines published by the special interest group on NSAID hypersensitivity reactions of the European Academy of Allergy and Clinical Immunology [5]. After excluding cross-hypersensitive subjects, we verified in cases of confirmed SR how many were SNIUAA and SNIDHR.

The diagnosis of SR patients is often complex, not risk free and requires trained personnel and specific resources [24]. In this study, we first verified tolerance to ASA in order to exclude patients with cross hypersensitivity to
NSAIDs. Of the remaining cases, those with positive skin tests and/or BAT were confirmed as SR to metamizole, as reported previously by our group [23]. Cases with negative skin tests and BAT required a minimum history of 2 previous reactions after metamizole administration to be considered SR. Although in previous studies looking at cross hypersensitivity to NSAIDs at least 3 episodes were required [18], in SR we have considered 2 clear episodes to be sufficient, provided that clinical history was reliable. Those patients with both negative skin tests and BAT that reported only 1 reaction after metamizole administration and contraindications for DPT were excluded from this study. This could contribute to some bias in this study in terms of the sensitivity of the skin tests, particularly for those with immediate reactions.

Skin testing was positive in $62.04 \%$ of the cases tested. Of the remaining cases $(\mathrm{n}=52), 28 \%$ of SNIUAA cases could be identified by BAT. The overall sensitivity including both tests was therefore $72.26 \%$. Skin and in vitro tests have shown variable results in different studies [23, 2527]. For immediate reactions, Gamboa et al. [25] reported BAT sensitivity to be $42.3 \%$ and specificity $100 \%$. Similar results were observed in a later study by Gomez et al. [23], in which the sensitivity of BAT was $54.9 \%$ and the specificity $85.7 \%$, and $62 \%$ of patients had positive skin tests to metamizole. In this study, we cannot establish the overall sensitivity of the tests because we did not perform BAT with metamizole in all patients. The time interval between the reaction and the study can affect the outcome of the tests [23], as has been shown in subjects with immediate hypersensitivity reactions to $\beta$-lactams $[28,29]$. We found differences comparing the time interval between the reaction and the performance of the tests in those who were negative and those who were positive. Another factor to consider that may contribute to the low 
sensitivity of diagnostic tests is the incomplete characterization of the chemical structures of metamizole and its metabolites [30]. Four major metamizole metabolites have been described in the literature [31]; however, we recently demonstrated the presence of arachidonoyl metabolites in patients receiving metamizole [32], and additional metabolites, such as oxalic acid derivatives, have been reported elsewhere [33]. It cannot be ruled out that in some patients metamizole metabolites may contribute to hypersensitivity reactions.

Considering the underlying mechanism in patients with immediate SR to pyrazolone derivatives, evidence (basophil activation and skin test positivity) supports an IgE-mediated mechanism [7,23]. There are only a few experimental studies on the quantification of IgE antibodies, and no detailed studies have been carried out in this field [8-10]. For delayed reactions, positive delayed ID and/or patch tests to the culprit drug with a characteristic T-cell infiltrate have been reported [6,34-38]. Further evidence has been provided by in vitro cellular assays $[38,39]$.

In the case of $\beta$-lactams, the time interval between drug administration and the appearance of symptoms is considered crucial for evaluating allergic reactions [40]. The reactions to these drugs can be considered immediate or nonimmediate. The former are induced by an IgE-mediated response, whilst for the latter, there are some controversies as to the underlying mechanism, especially for those with a time interval from 1 to $24 \mathrm{~h}$ after drug intake [41]. It has been shown that for the so-called accelerated reactions to amoxicillin, which occur between 1 and $6 \mathrm{~h}$, the mechanism is not IgE dependent [13]. In fact, some evidence indicates that these reactions are T-cell mediated [12]. However, to our knowledge, this mechanism has not yet been studied for NSAIDs. In this study, by analyzing the time interval between metamizole administration and reaction onset, we observed that $13 \%$ of the patients had reactions 1-24 h after metamizole intake. When analyzing basophil activation in cases where the reaction occurred 1-8 $\mathrm{h}$ after metamizole administration, we did not find any positive response in a group of $8 \mathrm{pa}-$ tients tested, suggesting that an IgE mechanism is unlikely. The time interval between drug administration and the onset of the reaction may be related to the production of different, as yet unidentified, metabolites. Metamizole metabolism occurs rapidly following intake, and some of the resultant metabolites are measurable in serum, urine and other biological fluids shortly after administration $[42,43]$.

Metamizole has more than 20 known metabolites [31] formed by either alkaline hydrolysis or biotransformation, but only a few studies have analyzed their immunogenic potential $[8,44]$. The identification of the adequate metabolite may be necessary to identify the underlying mechanisms and better diagnose these patients.

The percentage of atopy is high in these patients, but less than for cross-hypersensitive patients [20]. Atopy prevalence was similar in both SNIUAA and SNIDHR; however, more SNIDHR cases are needed to confirm our results.

In summary, we conclude that pyrazolones contribute to the production of selective reactions to NSAIDs, of which most are immediate. Although skin tests and BAT may aid in the diagnosis of these reactions, further research is needed to help identify the culprit metabolite and develop better diagnostic tools. To our knowledge, this is the largest study of cases with allergic responses to pyrazolones to date.

\section{Acknowledgments}

The present study has been supported by the Institute of Health 'Carlos III' of the Ministry of Economy and Competitiveness [grants cofounded by European Regional Development Fund (ERDF), Red de Reacciones Adversas a Alergenos y Farmacos (RD12/0013/0001 and PI15/01317)] and by Consejeria de Salud de la Junta de Andalucía (PI-0463-2013).

\section{Disclosure Statement}

None of the authors has any conflict of interest.

\section{References}

1 Thong BY, Tan TC: Epidemiology and risk factors for drug allergy. Br J Clin Pharmacol 2011;71:684-700

2 Dona I, Blanca-Lopez N, Torres MJ, GarciaCampos J, Garcia-Nunez I, Gomez F, et al: Drug hypersensitivity reactions: response patterns, drug involved, and temporal variations in a large series of patients. J Investig Allergol Clin Immunol 2012;22:363-371.
3 Zambonino MA, Torres MJ, Munoz C, Requena G, Mayorga C, Posadas T, et al: Drug provocation tests in the diagnosis of hypersensitivity reactions to non-steroidal anti-inflammatory drugs in children. Pediatr Allergy Immunol 2013;24:151-159.

4 Antunez C, Martin E, Cornejo-Garcia JA, Blanca-Lopez N, R-Pena R, Mayorga C, et al: Immediate hypersensitivity reactions to penicillins and other betalactams. Curr Pharm Des 2006;12:3327-3333. 
5 Kowalski ML, Asero R, Bavbek S, Blanca M, Blanca-Lopez N, Bochenek G, et al: Classification and practical approach to the diagnosis and management of hypersensitivity to nonsteroidal anti-inflammatory drugs. Allergy 2013;68:1219-1232.

6 Canto MG, Andreu I, Fernandez J, Blanca M: Selective immediate hypersensitivity reactions to NSAIDs. Curr Opin Allergy Clin Immunol 2009;9:293-297.

7 Himly M, Jahn-Schmid B, Pittertschatscher K, Bohle B, Grubmayr K, Ferreira F, et al: IgEmediated immediate-type hypersensitivity to the pyrazolone drug propyphenazone. J Allergy Clin Immunol 2003;111:882-888.

8 Schneider CH, Kasper MF, de Weck AL, Rolli H, Angst BD: Diagnosis of antibody-mediated drug allergy. Pyrazolinone and pyrazolidinedione cross-reactivity relationships. Allergy 1987;42:597-603.

9 Blanca M, Perez E, Garcia JJ, Miranda A, Terrados S, Vega JM, et al: Angioedema and IgE antibodies to aspirin: a case report. Ann Allergy 1989;62:295-298.

10 Harrer A, Lang R, Grims R, Braitsch M, Hawranek T, Aberer W, et al: Diclofenac hypersensitivity: antibody responses to the parent drug and relevant metabolites. PLoS One 2010;5:e13707.

11 Posadas SJ, Padial A, Torres MJ, Mayorga C, Leyva L, Sanchez E, et al: Delayed reactions to drugs show levels of perforin, granzyme B, and Fas- $\mathrm{L}$ to be related to disease severity. J Allergy Clin Immunol 2002;109:155-161.

12 Gomez E, Blanca-Lopez N, Salas M, Canto G, Campo P, Torres MJ, et al: Induction of accelerated reactions to amoxicillin by $\mathrm{T}$-cell effector mechanisms. Ann Allergy Asthma Immunol 2013;110:267-273.

13 Terrados S, Blanca M, Garcia J, Vega J, Torres MJ, Carmona MJ, et al: Nonimmediate reactions to betalactams: prevalence and role of the different penicillins. Allergy 1995;50:563567.

14 Szczeklik A, Stevenson DD: Aspirin-induced asthma: advances in pathogenesis, diagnosis, and management. J Allergy Clin Immunol 2003;111:913-921.

15 Jenkins C, Costello J, Hodge L: Systematic review of prevalence of aspirin induced asthma and its implications for clinical practice. BMJ 2004;328:434

16 Szczeklik A, Sladek K, Dworski R, Nizankowska E, Soja J, Sheller J, et al: Bronchial aspirin challenge causes specific eicosanoid response in aspirin-sensitive asthmatics. Am J Respir Crit Care Med 1996;154:1608-1614.

17 Sanak M, Gielicz A, Bochenek G, Kaszuba M, Nizankowska-Mogilnicka E, Szczeklik A: Targeted eicosanoid lipidomics of exhaled breath condensate provide a distinct pattern in the aspirin-intolerant asthma phenotype. J Allergy Clin Immunol 2011;127:1141.e21147.e2.

18 Blanca-Lopez N, J Torres M, Dona I, Campo $\mathrm{P}$, Rondon C, Seoane Reula ME, et al: Value of the clinical history in the diagnosis of urti- caria/angioedema induced by NSAIDs with cross-intolerance. Clin Exp Allergy 2013;43: 85-91.

19 Dona I, Blanca-Lopez N, Torres MJ, Gomez F, Fernandez J, Zambonino MA, et al: NSAIDinduced urticaria/angioedema does not evolve into chronic urticaria: a 12-year follow-up study. Allergy 2014;69:438-444.

20 Dona I, Blanca-Lopez N, Cornejo-Garcia JA, Torres MJ, Laguna JJ, Fernandez J, et al: Characteristics of subjects experiencing hypersensitivity to non-steroidal anti-inflammatory drugs: patterns of response. Clin Exp Allergy 2011;41:86-95.

21 Kowalski ML, Woszczek G, Bienkiewicz B, Mis M: Association of pyrazolone drug hypersensitivity with HLA-DQ and DR antigens. Clin Exp Allergy 1998;28:1153-1158.

22 Brockow K, Romano A, Blanca M, Ring J, Pichler W, Demoly P: General considerations for skin test procedures in the diagnosis of drug hypersensitivity. Allergy 2002;57:45-51.

23 Gomez E, Blanca-Lopez N, Torres MJ, Requena G, Rondon C, Canto G, et al: Immunoglobulin E-mediated immediate allergic reactions to dipyrone: value of basophil activation test in the identification of patients. Clin Exp Allergy 2009;39:1217-1224.

24 Ayuso P, Blanca-Lopez N, Dona I, Torres MJ, Gueant-Rodriguez RM, Canto G, et al: Advanced phenotyping in hypersensitivity drug reactions to NSAIDs. Clin Exp Allergy 2013; 43:1097-1109.

25 Gamboa PM, Sanz ML, Caballero MR, Antepara I, Urrutia I, Jauregui I, et al: Use of CD63 expression as a marker of in vitro basophil activation and leukotriene determination in metamizol allergic patients. Allergy 2003;58: 312-317.

26 Gamboa P, Sanz ML, Caballero MR, Urrutia I, Antepara I, Esparza R, et al: The flow-cytometric determination of basophil activation induced by aspirin and other non-steroidal anti-inflammatory drugs (NSAIDs) is useful for in vitro diagnosis of the NSAID hypersensitivity syndrome. Clin Exp Allergy 2004;34: 1448-1457.

27 Sanz ML, Gamboa P, de Weck AL: A new combined test with flowcytometric basophil activation and determination of sulfidoleukotrienes is useful for in vitro diagnosis of hypersensitivity to aspirin and other nonsteroidal anti-inflammatory drugs. Int Arch Allergy Immunol 2005;136:58-72.

28 Blanca M, Torres MJ, Garcia JJ, Romano A, Mayorga C, de Ramon E, et al: Natural evolution of skin test sensitivity in patients allergic to beta-lactam antibiotics. J Allergy Clin Immunol 1999;103:918-924.

29 Fernandez TD, Torres MJ, Blanca-Lopez N, Rodriguez-Bada JL, Gomez E, Canto G, et al: Negativization rates of IgE radioimmunoassay and basophil activation test in immediate reactions to penicillins. Allergy 2009;64:242-248.

30 Ariza A, Fernandez TD, Dona I, Aranda A, Blanca-Lopez N, Melendez L, et al: Basophil activation after nonsteroidal anti-inflamma- tory drugs stimulation in patients with immediate hypersensitivity reactions to these drugs. Cytometry A 2014;85:400-407.

31 Levy M, Zylber-Katz E, Rosenkranz B: Clinical pharmacokinetics of dipyrone and its metabolites. Clin Pharmacokinet 1995;28:216234.

32 Martinez C, Andreu I, Amo G, Miranda MA, Esguevillas G, Torres MJ, et al: Gender and functional CYP2C and NAT2 polymorphisms determine the metabolic profile of metamizole. Biochem Pharmacol 2014;92: 457-466.

33 Wessel JC, Matyja M, Neugebauer M, Kiefer H, Daldrup T, Tarbah FA, et al: Characterization of oxalic acid derivatives as new metabolites of metamizol (dipyrone) in incubated hen's egg and human. Eur J Pharm Sci 2006; 28:15-25.

34 Ozkaya-Bayazit E, Bayazit H, Ozarmagan G: Drug related clinical pattern in fixed drug eruption. Eur J Dermatol 2000;10:288-291.

35 Gonzalo-Garijo MA, Perez-Calderon R, De Argila D, Rodriguez-Nevado I: Metamizoleinduced acute generalized exanthematous pustulosis. Contact Dermatitis 2003;49:47-48.

36 Borja JM, Galindo PA, Gomez E, Feo F: Delayed skin reactions to metamizol. Allergy 2003;58:84-85.

37 Dalmau J, Serra-Baldrich E, Roe E, Peramiquel L, Alomar A: Use of patch test in fixed drug eruption due to metamizole (Nolotil). Contact Dermatitis 2006;54:127-128.

38 Macias E, Ruiz A, Moreno E, Laffond E, Davila I, Lorente F: Usefulness of intradermal test and patch test in the diagnosis of nonimmediate reactions to metamizol. Allergy 2007; 62:1462-1464.

39 Romano A, Torres MJ, Castells M, Sanz ML, Blanca M: Diagnosis and management of drug hypersensitivity reactions. J Allergy Clin Immunol 2011;127(3 suppl):S67-S73.

40 Blanca M, Romano A, Torres MJ, Fernandez J, Mayorga C, Rodriguez J, et al: Update on the evaluation of hypersensitivity reactions to betalactams. Allergy 2009;64:183-193.

41 Bircher AJ, Scherer Hofmeier K: Drug hypersensitivity reactions: inconsistency in the use of the classification of immediate and nonimmediate reactions. J Allergy Clin Immunol 2012;129:263-264.

42 Agundez JA, Martinez C, Benitez J: Metabolism of aminopyrine and derivatives in man: in vivo study of monomorphic and polymorphic metabolic pathways. Xenobiotica 1995; 25:417-427.

43 Vlahov V, Badian M, Verho M, Bacracheva N: Pharmacokinetics of metamizol metabolites in healthy subjects after a single oral dose of metamizol sodium. Eur J Clin Pharmacol 1990;38:61-65.

44 Zhu D, Becker WM, Schulz KH, Schubeler K, Schlaak M: Detection of IgE antibodies specific for 1-phenyl-2,3-dimethyl-3-pyrazoline5-one by RAST: a serological diagnostic method for sensitivity to pyrazoline drugs. Asian Pac J Allergy Immunol 1992;10:95-101. 